\title{
UNIFORM CONTINUITY OF QUASICONFORMAL MAPPINGS AND CONFORMAL DEFORMATIONS
}

\author{
PEKKA KOSKELA AND TOMI NIEMINEN
}

\begin{abstract}
We prove that quasiconformal maps onto domains satisfying a suitable growth condition on the quasihyperbolic metric are uniformly continuous even when both domains are equipped with internal metric. The improvement over previous results is that the internal metric can be used also in the image domain. We also extend this result for conformal deformations of the euclidean metric on the unit ball of $\mathbb{R}^{n}$.
\end{abstract}

\section{INTRODUCTION}

It is well known that quasiconformal mappings between sufficiently nice domains of $\mathbb{R}^{n}$ are uniformly continuous. Here the "niceness" of the image domain is measured, for example, by the so-called quasihyperbolic boundary conditions. Recall that the quasihyperbolic distance $k_{\Omega}(x, y)$ between two points $x, y$ of a domain $\Omega \subsetneq \mathbb{R}^{n}$ is defined by

$$
\inf _{\gamma} \int_{\gamma} \frac{d s}{d(z, \partial \Omega)}
$$

where the infimum is taken over all rectifiable curves joining $x$ to $y$ in $\Omega$. Gehring and Martio [3] proved that if $f: \Omega^{\prime} \rightarrow \Omega$ is a quasiconformal mapping between domains $\Omega^{\prime}, \Omega \subsetneq \mathbb{R}^{n}, n \geq 2$, and if the image domain $\Omega$ satisfies the growth condition

$$
k_{\Omega}\left(x_{0}, x\right) \leq C \log \frac{\operatorname{dist}\left(x_{0}, \partial \Omega\right)}{\operatorname{dist}(x, \partial \Omega)}+C
$$

for all $x \in \Omega$ with some fixed point $x_{0} \in \Omega$, then $f$ is globally Hölder continuous provided that the source domain $\Omega^{\prime}$ satisfies an additional geometric condition.

The above result was extended by Hencl and Koskela [6] (also see [7] and [8] for intermediate results) in the following way. Suppose that $\Omega$ satisfies a quasihyperbolic boundary condition

$$
k_{\Omega}\left(x_{0}, x\right) \leq \phi\left(\frac{\operatorname{dist}\left(x_{0}, \partial \Omega\right)}{\operatorname{dist}(x, \partial \Omega)}\right)
$$

for all $x \in \Omega$ with some fixed point $x_{0} \in \Omega$, or equivalently

$$
\operatorname{dist}(x, \partial \Omega) \leq \operatorname{dist}\left(x_{0}, \partial \Omega\right) \varphi\left(k_{\Omega}\left(x_{0}, x\right)\right)
$$

Received by the editors April 19, 2007.

2000 Mathematics Subject Classification. Primary 30C65.

Key words and phrases. Quasiconformal mapping, conformal metric. 
for $\varphi(t)=1 / \phi^{-1}(t)$, where $\varphi:[0, \infty) \rightarrow(0, \infty)$ is a non-increasing function satisfying

$$
\int_{0}^{\infty} \varphi(t) d t<\infty
$$

Then each quasiconformal mapping $f: \Omega^{\prime} \rightarrow \Omega$ satisfies

$$
\left|f\left(x^{\prime}\right)-f\left(y^{\prime}\right)\right| \leq C \Phi\left(C \log \frac{C}{\operatorname{diam}\left[x^{\prime}, y^{\prime}\right]_{\Omega^{\prime}}}\right)
$$

for all $x^{\prime}, y^{\prime} \in \Omega^{\prime}$. Here

$$
\Phi(s)=\int_{s}^{\infty} \varphi(t) d t
$$

and $\left[x^{\prime}, y^{\prime}\right]_{\Omega^{\prime}}$ denotes a quasihyperbolic geodesic joining $x^{\prime}$ to $y^{\prime}$ in $\Omega^{\prime}$. Recall that such a geodesic exists for all points $x^{\prime}, y^{\prime} \in \Omega^{\prime}$; see [4]. It was also shown in [6] that the estimate on the modulus of continuity is essentially sharp.

In this note, we shall extend the previous result by establishing the following theorem.

Theorem 1.1. Let $\Omega^{\prime}, \Omega \subsetneq \mathbb{R}^{n}, n \geq 2$, be domains and assume that $\Omega$ satisfies (1.1) and that (1.2) holds. Then each quasiconformal mapping $f: \Omega^{\prime} \rightarrow \Omega$ satisfies

$$
\delta_{\Omega}\left(f\left(x^{\prime}\right), f\left(y^{\prime}\right)\right) \leq C \Phi\left(C \log \frac{C}{\operatorname{diam}\left[x^{\prime}, y^{\prime}\right]_{\Omega^{\prime}}}\right)
$$

for all $x^{\prime}, y^{\prime} \in \Omega^{\prime}$.

Here the internal distance $\delta_{\Omega}(x, y)$ is defined as the infimum of the lengths of curves in $\Omega$ joining $x$ to $y$. Observe that always

$$
\left|f\left(x^{\prime}\right)-f\left(y^{\prime}\right)\right| \leq \delta_{\Omega}\left(f\left(x^{\prime}\right), f\left(y^{\prime}\right)\right),
$$

and hence (1.4) is stronger than (1.3).

Note that one could use the internal metric $\delta_{\Omega^{\prime}}$ also in the source domain $\Omega^{\prime}$ provided that the Gehring-Hayman inequality (cf. [2])

$$
\operatorname{diam}\left[x^{\prime}, y^{\prime}\right]_{\Omega^{\prime}} \leq C \delta_{\Omega^{\prime}}\left(x^{\prime}, y^{\prime}\right)
$$

is satisfied for all $x^{\prime}, y^{\prime} \in \Omega^{\prime}$.

We shall also generalize the above result for conformal deformations of the euclidean metric on the unit ball $\mathbb{B}^{n}$ of euclidean space. This seems to be a natural setting for the problem at hand. Thus, given a continuous density $\rho: \mathbb{B}^{n} \rightarrow \mathbb{R}_{+}$, we define a conformal metric $d_{\rho}$ by setting

$$
\operatorname{length}_{\rho}(\gamma)=\int_{\gamma} \rho(z)|d z|
$$

for a curve $\gamma$ in $\mathbb{B}^{n}$, and

$$
d_{\rho}(x, y)=\inf _{\gamma} \operatorname{length}_{\rho}(\gamma) \text { for } x, y \in \mathbb{B}^{n},
$$

where the infimum is taken over all curves joining $x$ and $y$ in $\mathbb{B}^{n}$. We also define a measure $\mu_{\rho}$ by setting

$$
\mu_{\rho}(E)=\int_{E} \rho^{n} d m_{n} \quad \text { for a Borel set } E \subset \mathbb{B}^{n},
$$

where $m_{n}$ denotes the $n$-dimensional Lebesgue measure. 
Further, we assume that the density $\rho$ satisfies a Harnack inequality; i.e., there exists a constant $A \geq 1$ so that

$$
\frac{1}{A} \leq \frac{\rho(x)}{\rho(y)} \leq A
$$

whenever $x, y \in B\left(z, \frac{1}{2}(1-|z|)\right)$ for some $z \in \mathbb{B}^{n}$. We also assume that the density $\rho$ satisfies a volume growth condition: there exists a constant $B>0$ so that

$$
\mu_{\rho}\left(B_{\rho}(x, r)\right) \leq B r^{n} \quad \text { for all } x \in \mathbb{B}^{n}, r>0 .
$$

Here $B_{\rho}(x, r)$ denotes an open ball with center $x$ and radius $r$ in the metric $d_{\rho}$. The motivation for conformal metrics arises primarily from the theory of quasiconformal mappings. Recall that the average derivative

$$
a_{f}(x)=\left(\frac{1}{m_{n}\left(B_{x}\right)} \int_{B_{x}} J_{f} d m_{n}\right)^{1 / n}, \quad B_{x}=B\left(x, \frac{1}{2}(1-|x|)\right),
$$

of a quasiconformal mapping $f: \mathbb{B}^{n} \rightarrow \Omega$ is a prime example of a density satisfying the above conditions. Note however that not all conformal densities arise from a quasiconformal mapping; see [1] for more information and examples.

In addition to (1.2), we will also assume that

$$
\beta \varphi\left(\log \frac{1}{t}\right) \leq C \varphi\left(\log \frac{1}{\beta t}\right)
$$

for all $t, \beta<1$. Note that this technical assumption is harmless: It follows from the Harnack inequality that $\rho(x) \geq(1-|x|)^{C}$ for all $x \in \mathbb{B}^{n}$, and hence the function $\varphi(t)$ in (1.6) below cannot decrease asymptotically faster than $\exp (-C t)$.

In the setting of conformal metrics on the unit ball we establish the following theorem.

Theorem 1.2. Assume that

$$
r_{x} \leq \rho(0) \varphi\left(\log \frac{1}{1-|x|}\right)
$$

for all $x \in \mathbb{B}^{n}$ with a non-increasing function $\varphi$ satisfying (1.2) and (1.5). Then

$$
d_{\rho}(x, y) \leq C \Phi\left(C \log \frac{C}{|x-y|}\right)
$$

for all $x, y \in \mathbb{B}^{n}$.

Here we write $r_{x}=\rho(x)(1-|x|)$. Recall that this quantity is comparable to the $\rho$-distance of $x$ to the boundary; see [1, Proposition 6.2]. Also recall that $k_{\mathbb{B}^{n}}(0, x)=\log \frac{1}{1-|x|}$. Thus one observes that the assumption (1.6) is a natural counterpart of the quasihyperbolic boundary condition (1.1) in our more general setting.

Let us finally point out that the improvement obtained in Theorems 1.1 and 1.2 in comparison to previous results is significant even in the usual setting of (quasi)conformal mappings of (a subset of) $\mathbb{R}^{n}$. Indeed, in many situations it is more natural to equip the image domain $\Omega$ with the internal metric instead of the euclidean metric, i.e. to use Theorem 1.1 instead of the results in [6]. For example, suppose that we are given a domain $\Omega \subset \mathbb{R}^{n}$, a set $E^{\prime} \subset \partial \Omega$ and a quasiconformal mapping $f: \mathbb{B}^{n} \rightarrow \Omega$. It is natural to ask for an estimate for the size of the set $E \subset \partial \mathbb{B}^{n}$ for which the radial limit $f(\xi)$ exists and $f(\xi) \in E^{\prime}$ for all $\xi \in E$. If $\Omega$ satisfies a suitable growth condition on the quasihyperbolic metric, one can estimate 
the Hausdorff dimension of $E$ from below by using the uniform continuity of the mapping $f$ implied by Theorem 1.1 or [6, Theorem 1.1]. However, it is perhaps surprising that the usage of the internal metric instead of the euclidean metric in the image domain may lead to a significant improvement on this dimension estimate; see [9] for an example of such a situation.

\section{Proofs of the Results}

We will first prove Theorem 1.2 by indicating the modifications needed in the proof of [6, Theorem 1.2]. As in [6], we will denote by $C$ various positive constants that may depend on the given data $n, \varphi, A, B$ and $\rho(0)$. In the proof of Theorem 1.1 , the constants $C$ may also depend on $K, \operatorname{dist}\left(x_{0}, \partial \Omega\right)$ and $\operatorname{dist}\left(f^{-1}\left(x_{0}\right), \partial \Omega^{\prime}\right)$. These constants may vary from expression to expression as usual. We write $\mathcal{W}$ for the Whitney decomposition of the unit ball $\mathbb{B}^{n}$; i.e., $\mathcal{W}$ is a collection of closed dyadic cubes $Q \subset \mathbb{B}^{n}$ with pairwise disjoint interiors such that

$$
\bigcup_{Q \in \mathcal{W}} Q=\mathbb{B}^{n}
$$

and that $\operatorname{diam}(Q) \leq \operatorname{dist}(Q, \partial \Omega) \leq 4 \operatorname{diam}(Q)$ (see [10] for the existence of such a decomposition). For a positive integer $j$ we denote by $W_{j}$ the set

$$
W_{j}=\left\{Q \in \mathcal{W}: j-1 \leq k_{\mathbb{B}^{n}}\left(0, x_{Q}\right)<j\right\},
$$

where $x_{Q}$ is the center of $Q$. We also define $r_{Q}=r_{x_{Q}}=\rho\left(x_{Q}\right)\left(1-\left|x_{Q}\right|\right)$.

Lemma 2.1. Suppose that the density $\rho$ satisfies (1.6) and that $\varphi$ satisfies (1.2). Let $\gamma \subset \mathbb{B}^{n}$ be a hyperbolic geodesic. Then there is an integer $j \in \mathbb{N}$ and a cube $Q \in \mathcal{W}_{j}$ such that $Q \cap \gamma \neq \emptyset$ and

$$
\operatorname{length}_{\rho}(\gamma) \leq C \rho(0) \Phi(j / 2) .
$$

Proof. Let $j=\min \left\{i \in \mathbb{N}\right.$ : there is $Q \in \mathcal{W}_{i}$ such that $\left.Q \cap \gamma \neq \emptyset\right\}$. Since $\operatorname{diam}_{\rho}(Q)$ is comparable to $r_{Q}$ for each $Q \in \mathcal{W}$ by the Harnack inequality, we have by the Gehring-Hayman inequality [1, Theorem 3.1] and the assumption (1.6) that

$$
\begin{aligned}
\operatorname{length}_{\rho}(\gamma) & \leq \sum_{\{Q \in \mathcal{W}: Q \cap \gamma \neq \emptyset\}} \operatorname{diam}_{\rho}(Q) \\
& \leq C \sum_{\{Q \in \mathcal{W}: Q \cap \gamma \neq \emptyset\}} r_{Q} \\
& \leq C \rho(0) \sum_{\{Q \in \mathcal{W}: Q \cap \gamma \neq \emptyset\}} \varphi\left(k_{\mathbb{B}^{n}}\left(0, x_{Q}\right)\right) \\
& \leq C \rho(0) \sum_{i=j}^{\infty} M_{i} \varphi(i),
\end{aligned}
$$

where $M_{i}=\#\left\{Q \in \mathcal{W}_{i}: Q \cap \gamma \neq \emptyset\right\}$. It was shown in the proof of [6, Lemma 3.5] that

$$
\sum_{i=j}^{\infty} M_{i} \varphi(i) \leq C \int_{j / 2}^{\infty} \varphi(t) d t .
$$

The claim follows by combining (2.1) and (2.2). 
Note especially that Lemma 2.1 implies the boundedness of $\mathbb{B}^{n}$ in the metric $d_{\rho}$. Indeed, for any $x_{1}, x_{2} \in \mathbb{B}^{n}$ we have that

$$
d_{\rho}\left(x_{1}, x_{2}\right) \leq \operatorname{length}_{\rho}\left[x_{1}, x_{2}\right]_{\mathbb{B}^{n}} \leq C \rho(0) \Phi(0)<\infty
$$

because of the assumption (1.2).

Lemma 2.2. Suppose that the density $\rho$ satisfies (1.6) and $\varphi$ satisfies (1.2). Let $\gamma \subset \mathbb{B}^{n} \backslash Q_{0}$ be a hyperbolic geodesic not entirely contained in $\frac{3}{2} Q$ for any $Q \in \mathcal{W}$. Then

$$
\operatorname{cap}\left(\gamma, Q_{0} ; \mathbb{B}^{n}\right) \geq C\left(\Phi^{-1}\left(C \text { length }_{\rho}(\gamma)\right)\right)^{1-n} .
$$

Here we denote by $Q_{0}$ the Whitney cube containing the origin. By $\frac{3}{2} Q$ we mean a cube concentric with $Q$ and with diameter $\frac{3}{2} \operatorname{diam}(Q)$. The usual conformal capacity of a pair of disjoint sets $E, F \subset \mathbb{B}^{n}$ is denoted by $\operatorname{cap}\left(E, F ; \mathbb{B}^{n}\right)$; see [6] for the precise definition.

Proof. Let a function $u \in W_{\text {loc }}^{1, n}\left(\mathbb{B}^{n}\right)$ be a test function for the $n$-capacity for the pair $\left(Q_{0}, \gamma\right)$; i.e., $u: \mathbb{B}^{n} \rightarrow[0,1]$ is a continuous function with locally $n$-integrable first order distributional partial derivatives and $u(x)=1$ for all $x \in \gamma$ and $u(x)=0$ for all $x \in Q_{0}$. By Lemma 2.1 we find a sequence of adjacent cubes which joins $Q_{0}$ to $\gamma$, i.e. cubes $\left\{Q_{i}\right\}_{i=0}^{k}$ such that $\bar{Q}_{i} \cap \bar{Q}_{i+1} \neq \emptyset$ for all $i \in\{0, \ldots, k-1\}, Q_{k} \cap \gamma \neq \emptyset$ and

$$
k \leq C \Phi^{-1}\left(C \frac{\operatorname{length}_{\rho}(\gamma)}{\rho(0)}\right) \leq C \Phi^{-1}\left(C \operatorname{length}_{\rho}(\gamma)\right)
$$

We divide the proof into two cases according to whether $u_{Q_{k}}<1 / 2$ or $u_{Q_{k}} \geq$ $1 / 2$. In the former case we can find a subset $F \subset Q_{k}$ whose Hausdorff 1-content $H_{1}^{\infty}(F)$ is comparable to the diameter of $Q_{k}$ and for which $u(y) \leq 1 / 2$ for all $y \in F$. Since $\gamma$ intersects $Q_{k}$ but is not entirely contained in $\frac{3}{2} Q_{k}$, we deduce that $H_{1}^{\infty}\left(\gamma \cap \frac{3}{2} Q_{k}\right) \geq \operatorname{diam}\left(\gamma \cap \frac{3}{2} Q_{k}\right) \geq C \operatorname{diam}\left(Q_{k}\right)$. Thus we have two compact sets $F$ and $\gamma \cap \frac{3}{2} Q_{k}$ in the cube $\frac{3}{2} Q_{k}$, both of which have Hausdorff 1-content comparable from below to $\operatorname{diam}\left(Q_{k}\right)$. Then

$$
\int_{\mathbb{B}^{n}}|\nabla u(x)|^{n} d x \geq \int_{\frac{3}{2} Q_{k}}|\nabla u(x)|^{n} d x \geq C(n),
$$

(see e.g., the proof of [5, Theorem 5.9]). This implies (2.4) with suitable constants because of the uniform upper bound (2.3) for the $\rho$-length of $\gamma$ implied by Lemma 2.1.

It only remains to prove the claim in the case $u_{Q_{k}} \geq 1 / 2$. By comparing the averages of $u$ in the cubes $Q_{0}, \ldots, Q_{k}$ with the help of the inequality

$$
\int_{Q_{i} \cup Q_{i+1}}\left|u-u_{Q_{i} \cup Q_{i+1}}\right| \leq C\left(\int_{Q_{i} \cup Q_{i+1}}|\nabla u|^{n}\right)^{1 / n}
$$

and using Hölder's inequality we obtain (cf. the proof of [6, Theorem 1.3])

$$
1 \leq C\left(\int_{\mathbb{B}^{n}}|\nabla u(x)|^{n} d x\right)^{\frac{1}{n}} k^{\frac{n-1}{n}} .
$$

This inequality together with the estimate (2.5) gives us (2.4). 
Proof of Theorem 1.2. Let $x, y \in \mathbb{B}^{n}$ and let $\gamma$ be a hyperbolic geodesic connecting $x$ to $y$. Let us first consider the case that $\gamma$ is contained in $\frac{3}{2} Q$ for some $Q \in \mathcal{W}$. Observe that in this situation the geodesic $\gamma$ intersects only $C(n)$ Whitney cubes and, moreover,

$$
\rho(x) \leq C \rho\left(x_{Q}\right)
$$

for all $x \in \frac{3}{2} Q$ by the Harnack inequality. Hence we obtain by the assumptions (1.6) and (1.5) that

$$
\begin{aligned}
d_{\rho}(x, y) & \leq C \rho\left(x_{Q}\right) \operatorname{length}(\gamma) \\
& \leq C \frac{\operatorname{leng} \operatorname{th}(\gamma)}{1-\left|x_{Q}\right|} \rho(0) \varphi\left(\log \frac{1}{1-\left|x_{Q}\right|}\right) \\
& \leq C \varphi\left(\log \frac{e}{\operatorname{length}(\gamma)}\right) \\
& \leq C \int_{\left[\log \frac{1}{\operatorname{length}(\gamma)}, \log \frac{e}{\text { length }(\gamma)}\right]} \varphi(t) d t \\
& \leq C \Phi\left(\log \frac{1}{\operatorname{length}(\gamma)}\right),
\end{aligned}
$$

because $\varphi$ is non-increasing. This gives us the desired estimate $\operatorname{since} \operatorname{length}(\gamma) \leq$ $C|x-y|$.

Let us then suppose that $\gamma$ is not contained in $\frac{3}{2} Q$ for any $Q \in \mathcal{W}$. This means in particular that either $x$ or $y$ ( say $x$ ) is in $\mathbb{B}^{n} \backslash \frac{3}{2} Q_{0}$. Hence there is a constant $\delta>0$ depending only on $n$ such that $\operatorname{dist}\left(x, Q_{0}\right) \geq \delta \operatorname{diam}\left(Q_{0}\right)$.

One of the following holds: Either $\operatorname{diam}(\gamma) \geq \delta \operatorname{diam}\left(Q_{0}\right) / 2$ or $\operatorname{diam}(\gamma)<$ $\delta \operatorname{diam}\left(Q_{0}\right) / 2$. In the first case we obtain by Lemma 2.1 (or the inequality $(2.3)$ ) that

$$
\frac{\operatorname{length}_{\rho}(\gamma)}{\operatorname{diam}(\gamma)} \leq \frac{C}{\delta \operatorname{diam}\left(Q_{0}\right)}
$$

which gives us a modulus of continuity no worse than (1.4) (note that $\operatorname{diam}(\gamma) \leq$ $C|x-y|)$.

On the other hand, if $\operatorname{diam} \gamma<\delta \operatorname{diam}\left(Q_{0}\right) / 2$, then also

$$
\delta \operatorname{diam}\left(Q_{0}\right) / 2 \leq \operatorname{dist}\left(\gamma, Q_{0}\right),
$$

because $\delta \operatorname{diam}\left(Q_{0}\right) \leq \operatorname{dist}\left(x, Q_{0}\right)$. A basic estimate on the conformal capacity now implies

$$
\begin{aligned}
\operatorname{cap}\left(\gamma, Q_{0} ; \mathbb{B}^{n}\right) & \leq C(n)\left(\log \frac{\operatorname{dist}\left(\gamma, Q_{0}\right)}{\operatorname{diam} \gamma}\right)^{1-n} \\
& \leq C(n)\left(\log \frac{\delta \operatorname{diam}\left(Q_{0}\right)}{2 \operatorname{diam} \gamma}\right)^{1-n}
\end{aligned}
$$

where the latter inequality follows from (2.6). Lemma 2.2, on the other hand, implies the lower bound

$$
\operatorname{cap}\left(\gamma, Q_{0} ; \mathbb{B}^{n}\right) \geq C\left(\Phi^{-1}\left(C \text { length }_{\rho}(\gamma)\right)\right)^{1-n}
$$

for the capacity. By combining (2.7) and (2.8) we arrive at

$$
\Phi^{-1}\left(C \text { length }_{\rho}(\gamma)\right) \geq C(n) \log \frac{\delta \operatorname{diam}\left(Q_{0}\right)}{2 \operatorname{diam} \gamma} .
$$

The claim follows since $\operatorname{diam}(\gamma) \leq C|x-y|$. 
Proof of Theorem 1.1. The proof is similar to the one of Theorem 1.2 and thus we only indicate the important modifications needed. Let $x^{\prime}, y^{\prime} \in \Omega^{\prime}$ and $\gamma=\left[x^{\prime}, y^{\prime}\right]_{\Omega^{\prime}}$, and let $\mathcal{W}$ be a Whitney decomposition of $\Omega$. As a counterpart of Lemma 2.1, one first establishes

$$
\delta_{\Omega}\left(f\left(x^{\prime}\right), f\left(y^{\prime}\right)\right) \leq C \operatorname{dist}\left(x_{0}, \partial \Omega\right) \Phi(j / 2)
$$

with $j=\min \left\{i \in \mathbb{N}\right.$ : there is $Q \in \mathcal{W}_{i}$ such that $\left.Q \cap f(\gamma) \neq \emptyset\right\}$ simply by following the proof of [6, Lemma 3.5] and observing that

$$
\delta_{\Omega}\left(f\left(x^{\prime}\right), f\left(y^{\prime}\right)\right) \leq \sum_{\{Q \in \mathcal{W}: Q \cap f(\gamma) \neq \emptyset\}} \operatorname{diam}(Q) .
$$

Write $Q_{0}$ for the Whitney cube containing the point $x_{0}$. By using (2.9) together with the assumption (1.2) one then obtains the estimate

$$
\operatorname{cap}\left(f(\gamma), Q_{0} ; \Omega\right) \geq C\left(\Phi^{-1}\left(C \delta_{\Omega}\left(f\left(x^{\prime}\right), f\left(y^{\prime}\right)\right)\right)\right)^{1-n}
$$

with similar arguments as in the proof of Lemma 2.2 (note that the internal diameter of $\Omega$ is finite by (2.9)). In this setting one can apply a standard capacity estimate to obtain (2.10) even for $f(\gamma)$ contained in $\frac{3}{2} Q$ for some $Q \in \mathcal{W}$; see the proof of [8, Theorem 1.4].

For the final conclusions define $F^{\prime}=f^{-1}\left(Q_{0}\right)$ and $\tilde{F}=f^{-1}\left(\frac{3}{2} Q_{0}\right)$, and take $\delta=\delta(n, K)>0$ such that $\operatorname{dist}\left(x^{\prime}, F^{\prime}\right) \leq \delta \operatorname{diam} F^{\prime}$ implies $x^{\prime} \in \tilde{F}$. Since the situation is trivial in the case that $x^{\prime}, y^{\prime} \in \tilde{F}$, assume that $x^{\prime} \in \Omega^{\prime} \backslash \tilde{F}$, which implies $\delta \operatorname{diam} F^{\prime} \leq \operatorname{dist}\left(x^{\prime}, F^{\prime}\right)$.

If $\operatorname{diam} \gamma \geq \delta \operatorname{diam} F^{\prime} / 2$, then the claim follows from (2.9) and (1.2). On the other hand, if $\operatorname{diam} \gamma<\delta \operatorname{diam} F^{\prime} / 2$, then also

$$
\delta \operatorname{diam} F^{\prime} / 2 \leq \operatorname{dist}\left(\gamma, F^{\prime}\right)
$$

because $\delta \operatorname{diam} F^{\prime} \leq \operatorname{dist}\left(x^{\prime}, F^{\prime}\right)$. Combining this inequality with a standard capacity estimate gives

$$
\operatorname{cap}\left(\gamma, F^{\prime} ; \Omega^{\prime}\right) \leq C(n)\left(\log \frac{\delta \operatorname{diam}\left(F^{\prime}\right)}{2 \operatorname{diam} \gamma}\right)^{1-n} .
$$

Finally, one naturally uses the elementary property

$$
\operatorname{cap}\left(f(\gamma), Q_{0} ; \Omega\right) \leq K \operatorname{cap}\left(\gamma, f^{-1}\left(Q_{0}\right) ; \Omega^{\prime}\right)
$$

of the quasiconformal mapping $f$ in order to link the inequalities (2.11) and (2.10).

\section{REFERENCES}

1. M. Bonk, P. Koskela and S. Rohde, Conformal metrics on the unit ball in euclidean space, Proc. London Math. Soc. (3) 77 (1998), 635-664. MR1643421 (99f:30033)

2. F. W. Gehring and W. K. Hayman, An inequality in the theory of conformal mapping, J. Math. Pures Appl. (9) 41 (1962), 353-361. MR0148884 (26:6381)

3. F. W. Gehring and O. Martio, Lipschitz classes and quasiconformal mappings, Ann. Acad. Sci. Fenn. Ser. A I Math. 10 (1985), 203-219. MR802481 (87b:30029)

4. F. W. Gehring and B. Osgood, Uniform domains and the quasihyperbolic metric, J. Anal. Math. 36 (1979), 50-74. MR581801 (81k:30023)

5. J. Heinonen and P. Koskela, Quasiconformal maps in metric spaces with controlled geometry, Acta Math. 181 (1998), 1-61. MR1654771 (99j:30025)

6. S. Hencl and P. Koskela, Quasihyperbolic boundary conditions and capacity: Uniform continuity of quasiconformal mappings, J. Anal. Math. 96 (2005), 19-35. MR2177180 (2006h:30017)

7. D. A. Herron and P. Koskela, Conformal capacity and the quasihyperbolic metric, Indiana Univ. Math. J. 45 (2) (1996), 333-359. MR1414333 (97i:30060) 
8. P. Koskela, J. Onninen and J. T. Tyson, Quasihyperbolic boundary conditions and capacity: Hölder continuity of quasiconformal mappings, Comment. Math. Helv. 76 (2001), 416-435. MR1854692 (2002j:30026)

9. T. Nieminen, Conformal metrics and boundary accessibility, Preprint 339, Department of Mathematics and Statistics, University of Jyväskylä, 2007.

10. E. M. Stein, Singular integrals and differentiability properties of functions, Princeton: Princeton University Press, 1970. MR0290095 (44:7280)

Department of Mathematics, University of Jyväskylä, P.O. Box 35, FI-40014, Finland

E-mail address: pkoskela@maths.jyu.fi

Department of Mathematics, University of Jyväskylä, P.O. Box 35, FI-40014, Finland

E-mail address: tominiem@maths.jyu.fi 